\title{
Durchgängige Feature-ID von der 3D-CAD Kon- struktion bis zur Qualitätssicherung
}

\author{
Christopher Saal
}

Digital Manufacturing Technologies, Corporate Technology Siemens $A G$

\section{Abstract}

Shorter product cycle times, higher quality and the desire for an ever-increasing variety of variants adorn the flagships of today's manufacturing industry - while reducing product development costs. The most important challenge is the interaction between quality improvement and cost reduction. Previously unresolved interface deficits between product definition and quality inspection have led to considerable additional work in programming measuring machines and have impaired flexibility in the product development process. Therefore, this publication describes methods and approaches for resolving the deficiencies within the process chain and summarizes the most recent insights. In addition, the article describes the current deficient situation in a real CAD-CAQ/CMM process chain.

Keywords: CAD-CAQ/CMM process chain, Inspection, Feature, Product Manufacturing Information (PMI) 


\section{Aktueller Stand zum Datentransfer innerhalb der CAD-CAQ/CMM- Prozesskette}

Die Digitalisierung industrieller Entwicklungs-, Fertigungs- und Qualitätssicherungsprozesse hat mittlerweile in einer Vielzahl von Unternehmen Einzug gefunden und ist dort nicht mehr wegzudenken. Ausgehend von einem 3DCAD-Modell soll es möglich sein, nahezu alle produktionsbegleitenden Prozesse - von der Konstruktion bis zur Endabnahme beim Kunden - teil- oder vollautomatisiert zu lenken. Gerade im Bereich der computergestützten Qualitätssicherung (engl.: Computer Aided Quality - CAQ), speziell bei der Programmierung von Koordinatenmessgeräten (engl.: Coordinate Measuring Machine CMM) und den daraus abgeleiteten Messprotokollen, gibt es diesbezüglich bisher nicht ausgeschöpftes Optimierungspotential [1]. Neben konstruktiven und fertigungstechnischen Daten sollen auch qualitätsrelevante Zusatzinformationen direkt mit dem 3D-CAD-Modell geliefert und somit Kommunikationsprobleme, Unklarheiten sowie individuelle Fehlinterpretationen, bereits im Anfangsstadium der Produktdefinition, reduziert werden [2][3]. Die derzeit ungelösten arbeitsorganisatorischen und technologischen Herausforderungen sind auf Schnittstellenbarrieren zurückzuführen, die den Datentransfer zwischen der Konstruktions- und der Messgerätesoftware behindern (s. Bild 1). Die bestehende Entwicklungslücke stört die Durchgängigkeit der digitalen CADCAQ/CMM-Prozesskette und beeinträchtigt die Prozesstransparenz innerhalb der Produktentstehung.

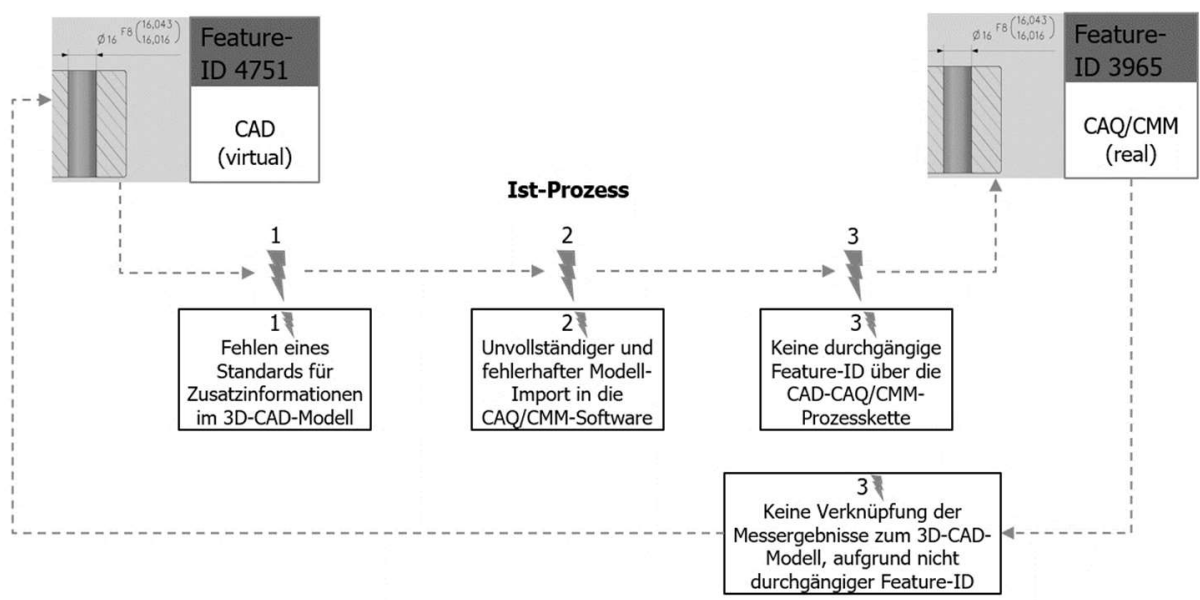

Bild 1: Schnittstellenbarrieren innerhalb der CAD-CAQ/CMM-Prozesskette 


\section{Werkzeuge zur Erreichung einer durchgängigen 3D-Prozesskette}

Obwohl sich der Einsatz 3D-fähiger-CAD-Systeme in Unternehmen in den letzten Jahren etabliert hat, wird für die Messgeräteprogrammierung sowie für die Messprotokollierung noch immer die aus dem 3D-CAD-Modell abgeleitete 2D-Zeichnung als führendes Dokument verwendet. Nicht-geometrische Informationen wie Maß-, Form- und Lagetoleranzen sowie Oberflächenangaben der zu prüfenden Elemente, werden manuell unter Zuhilfenahme verschiedener Ansichten und Schnitte auf der 2D-Zeichnung erzeugt, was arbeitsintensiv und fehleranfällig ist [4]. Zudem können Änderungen in der Produktdefinition so nur schwer nachvollzogen werden. Dieses Vorgehen soll zukünftig durch die Verwendung von Product Manufacturing Information (PMI), d.h. direkt mit dem 3D-CAD-Modell verknüpfte Informationen, abgelöst werden (s. Bild 2). Bislang ist es jedoch nicht möglich, die 3D-CAD-Modelle zusammen mit den Product Manufacturing Information vollständig und fehlerfrei in die CAQ/CMM-Software der Messgeräte zu importieren und eine eindeutige Zuordnung der Prüfmerkmale zu den jeweiligen Konstruktionselementen (Features) über die komplette CAD-CAQ/CMM-Prozesskette hinweg zu gewährleisten [5]. Umgekehrt lassen sich prüftechnisch ermittelte Sollwert-Abweichungen aus der Qualitätssicherung nicht direkt, d.h. ohne individuelle, menschliche Interpretation sowie Nach- bzw. Mehrarbeit, dem entsprechenden Konstruktionselement zuordnen. Ursache hierfür ist das Fehlen standardisierter Dateiformate, Normen sowie funktionaler Schnittstellenlösungen zwischen den 3D-CAD-Systemen und den CAQ/CMM-Systemen, die über den reinen Geometrieaustausch hinaus auch PMI vollumfänglich unterstützen [1].

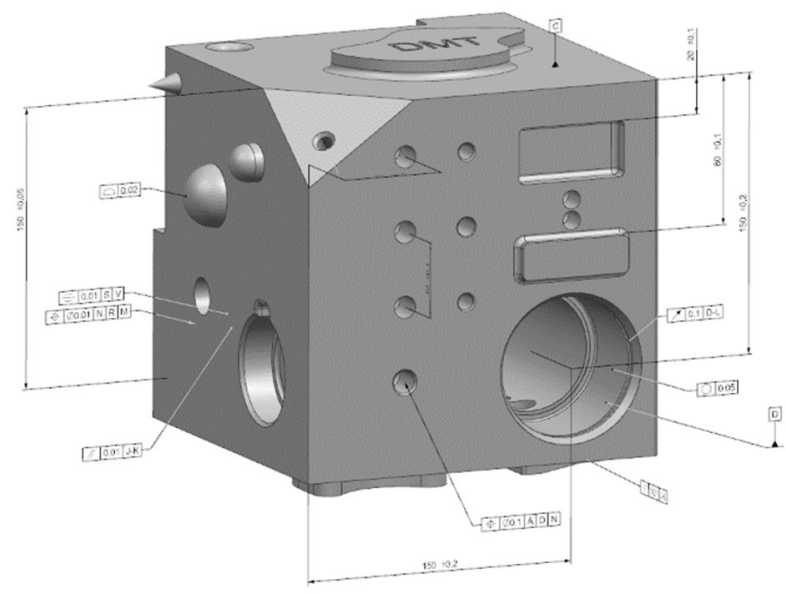

Bild 2: Product Manufacturing Information (PMI) am 3D-CAD-Testmodell 
Nach Hehenberger [6] umfasst die 3D-Prozesskette sechs Bereiche - von der Produktentwicklung bis zur Qualitätskontrolle - die von verschiedenen anwendungsspezifischen CAx-Systemen (Computer Aided $\mathrm{x}$ ) unterstützt werden (s. Bild 3). Der Einsatz der CAx-Systeme wird mit der Integration getrennter Funktionsbereiche und der Schaffung eines durchgängigen Informationsflusses zwischen den unterschiedlichen Teilbereichen in einem Unternehmen begründet [7]. Neben dem CAD (Computer Aided Design) im konstruktiven sowie dem CAM (Computer Aided Manufacturing) im fertigungstechnischen Bereich haben sich im Qualitätssektor die CAQ-Systeme (Computer Aided Quality) etabliert. CAQ beinhaltet neben der Messdatenprotokollierung, auch die Programmierung der Koordinatenmessgeräte und wird deshalb als „Bindeglied zwischen virtueller und realer Welt" verstanden [8]. Hier werden die realen Messdaten des gefertigten Produktes mit den virtuellen 3D-CAD-Daten verglichen und ausgewertet.

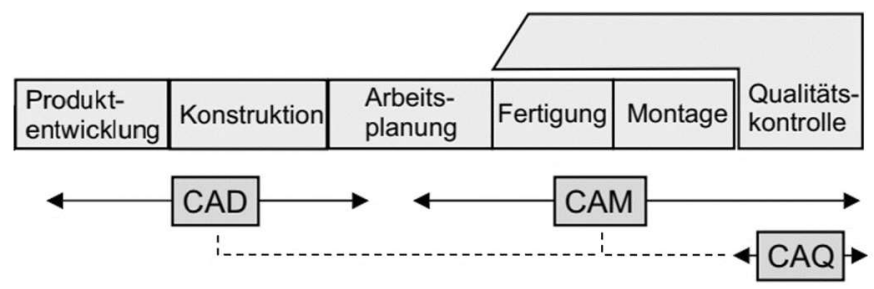

Bild 3: CAx-Systeme der rechnergestützten 3D-Prozesskette nach [6]

Um eine durchgängige 3D-Prozesskette zu erreichen, ist eine Verschmelzung der einzelnen Teilbereiche inklusive ihrer CAx-Methoden notwendig. Jedoch ist die Umsetzung, speziell auf den organisatorischen und informationstechnischen Ebenen, mit unterschiedlichen Herausforderungen behaftet. Hierzu zählen unter anderem der Umgang mit internen Datenzugriffsrechten sowie die Einführung und Integration von Schnittstellen- und Datenbanklösungen für den Datentransfer innerhalb eines Unternehmens [7]. Sogenannte „Features" stellen einen Lösungsansatz dar und sollen zur Beschleunigung der Systemkompatibilität beitragen. Der Feature-Begriff ist jedoch bislang nicht eindeutig definiert [9]. Neben der Beschreibung von kompletten Bauteilen, Informationseinheiten sowie Gestalt-, Konstruktions- und Fertigungselementen wird ein Feature auch als informationstechnisches Element verstanden, das in Gestalt eines Formfeatures sowohl Flächen, Konturen als auch Volumen darstellen kann [10] [11] [12]. Die für diese Forschungsarbeit geeignetste Definition beschreibt ein Feature als Geometrieelement, das zusätzlich zu seinen geometrischen Daten auch nichtgeometrische Informationen aufnehmen kann [9]. Dabei bilden logische Verknüpfungen von Formelementen, die zur Beschreibung von Design und

4 
Geometrie vorgesehen sind, die Konstruktionsabsicht ab. Sie werden als Konstruktions-, Prozess-, Fertigungs- und Informationsfeatures in verschiedenen Teilbereichen der Produktentstehung angewendet [9].

In Bezug auf den durchgängigen und eindeutigen ID- sowie Datentransfer vom 3D-CAD-System ins CAQ/CMM-System sind besonders die Bedeutung und die Eigenschaften der Konstruktionsfeatures näher zu betrachten. Das Konstruktionsfeature ist ein geometrisches Konstruktionselement, das zum einen in Geometriefeature und zum anderen in Designfeature unterschieden wird. Geometriefeatures umfassen beispielsweise Koordinatensysteme, Achsen, Ebenen und Volumen, wohingegen Designfeatures Rundungen, Fasen, Einstiche und Materialschnitte beschreiben (s. Bild 4). [9]

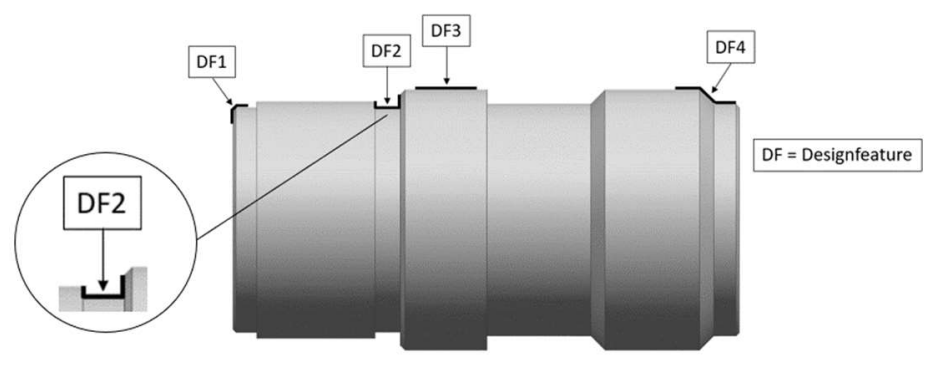

Bild 4: Unterschiedliche Designfeatures am Beispiel einer Welle

Beim Feature-Based-Modeling werden genau diese Eigenschaften und $\mathrm{Zu}$ sammenhänge genutzt. Dabei werden auf Basis Boolescher Operationen Geometrieelemente aus Instanzen generischer Grundkörper im 3D-CAD-System erzeugt und nachfolgend wieder zu einem Designfeature zusammengefasst. Demzufolge ist es möglich, dass ein Designfeature, aufgrund der im 3D-CADSystem hinterlegten Modellierungstechnik, aus mehreren einzelnen Flächen besteht und das zu tolerierende Element beschreibt. Das Designfeature DF2 aus Bild 4 besteht demnach aus den drei hervorgehobenen Flächen. Diese Flächen werden innerhalb eines Arbeitsschrittes in der Fertigung erzeugt und bilden die Referenzflächen für die Tolerierung in der Produktdefinition. Informationsfeatures, wie beispielsweise die PMI, können auf entsprechende Designfeatures verlinkt werden und so innerhalb der Produktdefinition wichtige Zusatzinformationen direkt mitliefern [13]. Diese Kopplung bildet die Grundlage für die nachfolgende Vorgehensweise zur Methodenfindung und wird für den weiteren Forschungsverlauf sowie anschließend im experimentellen Teil erneut aufgegriffen. 


\section{Methode und Vorgehensweise zur Entwicklung einer durchgängigen 3D-Prozesskette}

Zur Entwicklung eines herstellerneutralen Standards für eine durchgängige Feature-ID von der 3D-CAD Konstruktion bis zur Qualitätssicherung sind zunächst die Strukturen der zur Anwendung kommenden Methoden der 3D-CADund CAQ/CMM-Systeme zu analysieren. Anschließend sind Testmodelle in den 3D-CAD-Systemen aufzubauen und mit Zusatzinformationen in Form von PMI zu versehen, die die notwendigen Prüfmerkmale und Toleranzangaben beinhalten. Auf Basis der Feature-basierten Modellierung werden die Informationsfeatures (PMI) direkt mit den Designfeatures verlinkt. Außerdem sind die geltenden Regeln und Richtlinien bestehender Normen [14] zu berücksichtigen, um reproduzierbare Erkenntnisse für einen einheitlichen ID-Transfer vom 3D-CADSystem zum CAQ/CMM-System zu generieren.

Noch vor dem Import der 3D-CAD-Modelle ins CAQ/CMM-System sind die nativen Dateiformate (.prt, .asm, etc.) der Testmodelle in unterschiedliche neutrale Austauschformate (.jt, .stp, etc.) zu exportieren, um die Basis für einen systemübergreifenden Datenaustausch zu schaffen. Hierbei wird untersucht, welche Informationen beim Export vom nativen ins neutrale Datenformat übertragen werden und welche Daten bereits bei der Formatumwandlung im Konstruktionsumfeld verloren gehen (s. Bild 5). Somit können auftretende Problemstellen innerhalb der CAD-CAQ/CMM-Prozesskette vorab lokalisiert und mögliche Fehlerursachen ausgeschlossen werden, ohne dass überhaupt ein Datenimport vom 3D-CAD-System ins CAQ/CMM-System stattgefunden hat.

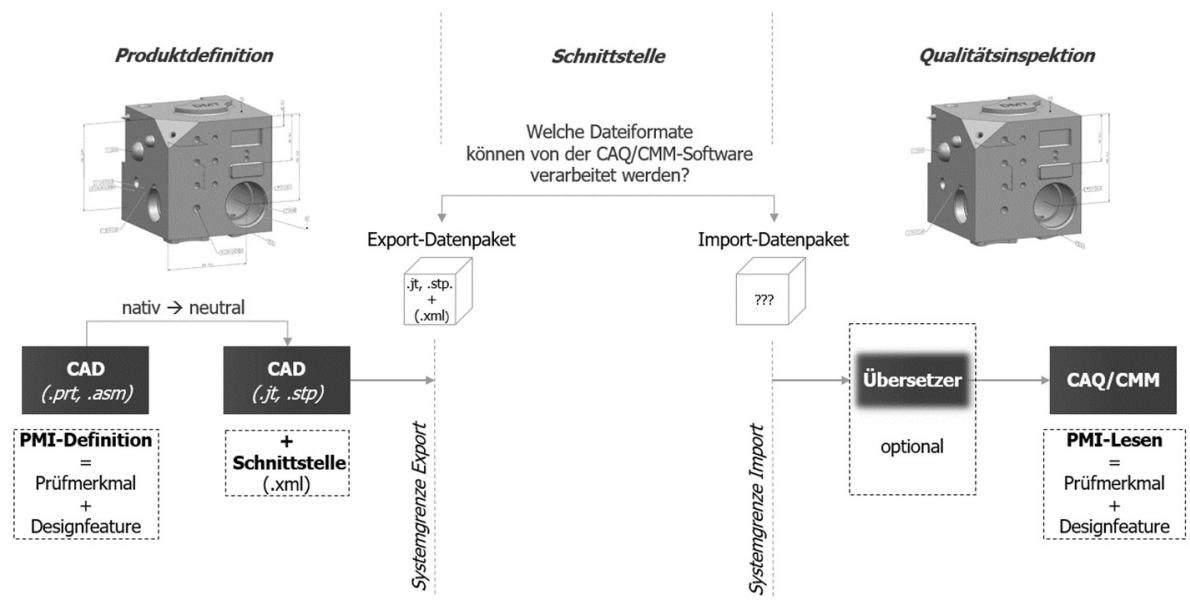

Bild 5: Datentransfer innerhalb des CAD-CAQ/CMM-Prozesses

6

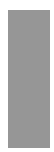


Sind alle für den Datenaustausch notwendigen Vorbereitungen innerhalb der Produktdefinition abgeschlossen, wird die Übertragung der neutralen Modelldaten vollzogen. Mittels der im Vorfeld durchgeführten Systemstrukturanalyse kann hier bereits vorab ermittelt werden, welche Dateiformate bzw. Datenpakete technisch überhaupt von den jeweiligen Messsoftwaresystemen verarbeitet werden können. Anschließend wird geprüft, ob und in welchem Umfang die Informationen aus dem neutralen 3D-CAD-Datenpaket beim Import ins CAQ/CMM-System übertragen werden. Die ermittelten Ergebnisse des Imports werden im weiteren Verlauf anhand vordefinierter Kriterien analysiert und bewertet. Folglich können neue Methoden und standardisierte Regeln für einen eindeutigen Feature-ID-Transfer vom 3D-CAD-System zum CAQ/CMM-System generiert bzw. abgeleitet werden. [15]

\section{Beispiele für derzeitige Herausforderungen und Defizite innerhalb einer realen, digitalen CAD-CAQ/CMM-Prozesskette}

Im Folgenden werden die Untersuchungsergebnisse bezüglich des Datentransfers zwischen Siemens NX 11 (3D-CAD-System) und Zeiss CALYPSO 2018 (CAQ/CMM-System) vorgestellt. Der modellierte Testwürfel aus Abschnitt 1, Bild 2 enthält eine Vielzahl von unterschiedlichen Konstruktions- und Geometrieelementen, die es ermöglichen nahezu alle nach Norm festgeschriebenen Maß-, Form- und Lagetoleranzen sowie einzelne Sonderformen in Bezug auf den durchgängigen PMI- und Datentransfer zu testen. Die PMI sind nach den, in ISO 16972 [14] definierten, Richtlinien angelegt und direkt auf die zugehörigen Designfeatures verlinkt. Insgesamt enthält das Testmodell 106 verschiedene Tolerierungsvariationen, wie beispielsweise Ebenheiten oder Parallelitäten, die mithilfe der PMI-Methode am 3D-CAD-Modell in die Messsoftware der Fa. Zeiss übertragen werden. Hierzu wird zunächst die native CAD-Datei (.prt) aus NX über die CAD-Schnittstelle in CALYPSO geladen. Bei diesem Test werden 35 der 106 Product Manufacturing Information fehlerhaft, unvollständig oder erst gar nicht in die Ziel-Software importiert. Die Übertragungslücken sind auf unterschiedliche Ursachen zurückzuführen, die nachfolgend zusammengefasst werden. Es ist anzumerken, dass aufgrund des inhaltlichen Rahmens dieses Papers nur die Problemstellen beschrieben werden, die auf Aspekte des Feature-Based-Modeling sowie der PMI-Definition zurückzuführen sind. Grundlegend fehlende Plug-ins innerhalb der Messsoftware werden nicht weiter behandelt.

In erster Linie sind Bemaßungs- und Bezugsinformationen am 3D-CAD-Modell von Datenübertragungsproblemen betroffen. Beispielsweise werden Fasenund Durchmesserbemaßungen, die in NX definiert sind, in CALYPSO nicht erkannt. Insbesondere Durchmesserangaben über Schnittkurven und 
Zylinderflächen sind hiervon betroffen. CALYPSO erzeugt beim Import einen internen Systemfehler, da ein Teil der im 3D-CAD-Modell referenzierten und verlinkten Flächen nicht eindeutig zugeordnet werden. Zudem werden bei Durchmesserbemaßungen über mehrere Zylindersegmente in CALYPSO für jedes Segment separate Prüfmerkmale angelegt, obwohl in NX lediglich ein PMI für die vorhandenen Zylindersegmente definiert ist (s. Bild 6). Auch können Lochkreisdurchmesser über Bohrungsmuster im 3D-CAD-System in CALYPSO nicht verarbeitet werden.
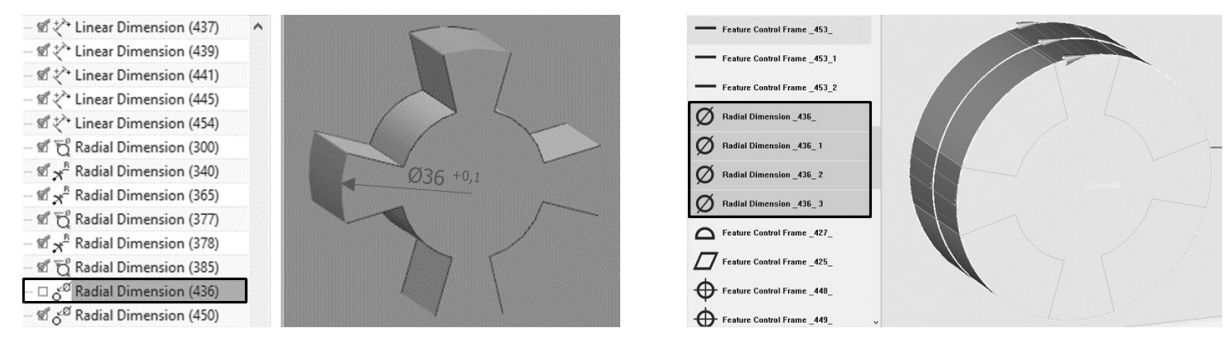

Bild 6: Durchmesserbemaßung über mehrere Zylindersegmente - PMI-Definition in Siemens NX (links) und PMI-Verarbeitung in Zeiss CALYPSO (rechts)

Bei der Übertragung von Einzelbezügen mittels der PMI-Methode aus NX, weist die getestete Messsoftware ebenso einige Defizite auf. Beispielsweise werden Kugelflächen aus NX in der CAQ/CMM-Software teils als Freiformfläche und teils als Kugel interpretiert. Des Weiteren wird bei gemeinsamen Bezügen nur das erste Bezugselement beim Modellimport eingelesen, anstatt der Erstellung eines gemeinsamen Bezugselements. Das zweite Bezugselement wird in Zeiss CALYPSO nicht erkannt und folglich nicht weiter berücksichtigt. Auch fordert CALYPSO zwingend Toleranz- und Bemaßungsbezüge, obwohl keine Bezüge im 3D-CAD-Modell definiert sind und die Toleranz per Definition lediglich für das Geometrieelement selbst ausgewertet werden soll.

Weitere Untersuchungen zeigen unterschiedliche Darstellungen der PMI im Strukturbaum der beiden Software-Systeme, da CALYPSO beim Import nicht die Feature-Reihenfolge aus NX übernimmt, sondern die strukturelle Anordnung der PMI auf Basis einer systeminternen, zeitoptimierten Messprogrammerstellung erzeugt. Die unterschiedlichen Darstellungsweisen erschweren nicht nur die Reproduktion der im 3D-CAD-Modell enthaltenen Informationen, sondern sorgen auch für einen erhöhten Zeitaufwand bei einer möglichen Fehleranalyse. Auch die IDs der tolerierten und in NX modellierten Designfeatures bleiben in CALYPSO unbeachtet. Lediglich die ID der PMI wird eindeutig beim Import in die Messsoftware übertragen. Die Möglichkeit auf direkte 
Rückschlüsse vom Messprotokoll auf die Produktdefinition oder Fertigung ist somit nicht gegeben und beeinträchtigt den durchgängigen Informations- und Datentransfer innerhalb der 3D-Prozesskette.

\section{Diskussion und weiteres Vorgehen}

Die bisherigen Erkenntnisse bestätigen die, aus der Literatur entnommenen und vorab beschriebenen, Problemstellen innerhalb der CAD-CAQ/CMM-Prozesskette. Fehlende, teils unzureichende Standards bereits in der Produktdefinition sowie vorhandene Schnittstellendefizite, stören die Durchgängigkeit der 3D-Prozesskette und verstärken die Nutzungshemmungen der PMI-Methode im 3D-CAD-Modell, sodass die 2D-Zeichnung noch immer als führendes Dokument aus dem 3D-CAD-Modell für Fertigungs- und Qualitätssicherungsprozesse abgeleitet wird. Das Ziel eines führenden und funktionalen 3D-CAD-Modells (engl.: Functional Information Model - FIM) zur Ablösung der 2D-Zeichnung bleibt somit bisher unerreicht. Um die Akzeptanz der PMI-Nutzung und somit auch die der Digitalisierung in Unternehmen zu fördern, sind neue Methoden für einen systemkompatiblen Datentransfer zu entwickeln. Hierzu sind weitere Software-Systeme, Dateiformate und Schnittstellen nach den in Abschnitt 3 beschriebenen Methoden zu untersuchen, die besonders auf die Entwicklung einer durchgängigen Feature-ID abzielen, um konstruktive, fertigungstechnische und qualitätsspezifische Prozesse in Unternehmen direkt verknüpfen und eindeutig untereinander zuordnen zu können. Auftretende Parallelen und Überschneidungen aus den Forschungsergebnissen können gezielt genutzt und neue Standards, die die bisherigen Normen und Richtlinien ergänzen oder gänzlich ersetzen, abgeleitet werden.

\section{Literatur}

[1] Kaubisch, F.: "3D-CAD- und NCM-Daten zur Steuerung von Messgeräten", Morsak-Verlag, Grafenau, 2015.

[2] Alemanni, M.; Destefanis, F.; Vezzetti, E.: "Model-based definition design in the product lifecycle management scenario", The International Journal of Advanced Manufacturing Technology 52, 2011, 1-14.

[3] Liu, R.; Duan, G.; Liu, J.: "A framework for model-based integrated inspection", The International Journal of Advanced Manufacturing Technology 103, 2019, 1-23.

[4] Neubert, S.; Robl, P.: "3D-CAD-Modelle lenken die Fertigung", VDI-ZIntegrierte Produktion, Düsseldorf, 2018. 
[5] Lipman, R.; Lubell, J.: "Conformance checking of PMI representation in CAD model STEP data exchange files", Computer Aided Design 66, 2015, 14-23.

[6] Hehenberger, P.: "Computerunterstützte Fertigung", Springer-Verlag, Berlin Heidelberg, 2011.

[7] Vajna, S. et al.: "CAx für Ingenieure - Eine praxisbezogene Einführung", Springer-Verlag, Berlin Heidelberg, 2009.

[8] Inkamp, D.: "Digitalisierung und Virtualisierung für die Dimensionelle Messtechnik", Carl Zeiss IMT GmbH, Oberkochen, 2018.

[9] Robl, P.: "Integratives NC-Modell für die Vereinfachung der werksübergreifenden Produktion auf der Basis einer Feature-Skelett-Methode", Morsak-Verlag, Grafenau, 2013.

[10] Weber, C.; Krause, F.: "Features mit System - die neue Richtlinie VDI 2218", VDI-Bericht 1497, 1999, 43-76.

[11] Krause, F.; Lehmann, C.: "Erweiterung des rechnergestützten Konstruierens durch Wissensverarbeitung", VDI-Bericht 775, 1989.

[12] Weber, C.: "What is a feature and what is its use? - Results of FEMEX working group I", Proceedings of the $29^{\text {th }}$ International symposium on automotive technology and automation, 1996, 287-296.

[13] Vajna, S.; Podehl, G.: "Durchgängige Produktmodellierung mit Features", CAD-CAM Report 3, 1998, 1-8.

[14] International Organization for Standardization (ISO): "Technical product documentation - Digital product definition data practices", ISO 16792, ISO-Norm, 2015.

[15] Lindemann, U.: "Methodische Entwicklung technischer Produkte: Methoden flexibel und situationsgerecht anwenden", Springer-Verlag, Berlin Heidelberg, 2009. 